\title{
Secure Cloud Computing Mechanism For ENHANCING: MTBAC
}

\author{
Payal Buha ${ }^{1}$, Priyanka Sharma ${ }^{2}$ \\ ${ }^{1}$ M.Tech (Cyber Security), \\ ${ }^{2}$ Professor (Information Technology)
${ }^{1,2}$ Raksha Shakti University, Ahmedabad,India
}

\begin{abstract}
The development of the cloud system, A large number of vendors can visit their users in the same platform directing their focus on the software rather than the underlying framework. This necessary require the distribution, storage analysis of the data on cloud accessing virtualized and scalable web services with broad application of cloud, the data security and access control become a major concern. The access to the cloud requires authorization as well as data accessibility permission. The verification and updation of data accessibility permissions and data must be done with proper knowledge which requires identification of correct updates and block listed users who are intruder to cloud Introducing the false data system. In this paper we approach to builds a mutual trust relationship between users and cloud for accessing control method in cloud computing environment focusing on the system integrity and its security. The proposed approach is executed as a procedure manner and includes many steps to identify the user's credibility in the cloud network.
\end{abstract}

\section{KEYWORDS}

MTBAC, ACO Algorithm, Access Control, Security, K-means Algorithm, Cloud Computing

\section{INTRODUCTION}

Access control mechanism has become important issue in cloud computing to ensure the security of resources and users updates on the cloud [1]. The user can make use of various cloud resources with the acceptance of the certificate from the authorization center for accessing the cloud [2].The traditional methods for access control were not able to solve the problems such as uncertainty and vulnerability to the attacks from the unauthorized or malicious users. Cloud Computing is distributed environment therefore anonymity of information are some basic features of cloud [3]. Hence, security in such cases become important for the data across the various sites and user on various cloud. Some of the major challenges in Cloud Computing such as: 1) Cost Reduction 2) Cloud Operation 3) Level of Service etc. The problem may be arising to approach this method is how to check for each anonymous user, the behavior user and the malicious user. It is also issues to authentication certificate for authorized user.

\section{OBJECTIVE}

Objective of this paper is that to implement the identification of each anonymous user by registration process. Once the user get the authentication certificate than the behavior of each user is tracked, while accessing the cloud resource this behavior information is stored in both user review and system review table in database. Based on this information the user is valid or 
blacklisted user is identified. In order to describe trust relationships among the multiple nodes providing cloud services, K- means cluster is applied for obtaining more similar relationship attributes to group together. As a result, a trust degree is established between similar interacted entities for accessing resources in the cloud environment. A further enhancement is established by applying population based intelligent search technique such as ant colony optimization approach for calculating optimum trust degree between more similar interacted entities with high credibility for accessing optimum resources and developing strong trust relationship between users and cloud service nodes.

\section{Motivation}

In this modern technology many organization either large or small use cloud computing today either directly or indirectly instead of traditional on site alternatives. By the end of 2015 it's estimated that $90 \%$ of businesses will be using at least one cloud service. Alongside this growth, Cloud Computing has also seen an increase in criminal activities in the cloud.

\section{RELATED WORK}

Access control is a mechanism which permits, refutes and hampers admission to a system. It may observe and maintain all tries made to enter in to the system. Access Control can isolate users bidding to access a system illegal. It is a mechanism which improves the security of Cloud Computing.

\subsection{Trust Base Access Control Model in Multi domain}

In May 2013, guoyunlin, YuyuBie and Min Lei proposed a trust base access control model in multi domain which combined with RBAC mechanism. They discuss local domain control policies in which access control policies include role assignment trust management authentication and authorization. They has also discuss cross domain policies which include role translation by using of means of role association and converted outer domain roles into local domain host.[4]

\subsection{Relation Between user and Relation between Access control permission}

In November 2011NurmamatHelil,Mucheol Kim and Sangyong Han have examine relation between users and relation between access control permission they have generalize the users according to their personal identity similarities and historical access interest similarities to improve flexibility of constraints of effectively[5].

\subsection{Trust Cloud Computing Platform}

In May 2009Nuno Santos presented the design of trust cloud computing platform that provide IaaS services like Amazon institute to provide cloud box execution environment. This platform guarantees confidential execution of guest virtual machine and allow user to attached the IaaS provider and check if service is secure or nor before launching the VM[6].

\subsection{Security Policies base trust TOrBAC}

In November 2012 Mustapha Ben Saidi and Abderrahim Marzouk proposed security policies base trust TOrBAC adapted to cloud and process of real time control policies violation. In this paper new ideas can review notification mechanism in critical information system. They have developed comprehensive model for application systems model for application systems wishing to integrate computing cloud [7]. 
International Journal of Information Sciences and Techniques (IJIST) Vol.6, No.1/2, March 2016

\begin{tabular}{|c|c|c|c|c|c|}
\hline Scenario & $\begin{array}{c}\text { Trust } \\
\text { and risk }\end{array}$ & Multi-Domain & TOrBAC & TCC-P & MTB-AC \\
\hline Confidentiality & $\begin{array}{c}\text { Very } \\
\text { good }\end{array}$ & Increase & Increase & Good & Increase \\
\hline Integrity & Increase & Increase & Very good & Increase & Very good \\
\hline Trust & Increase & Increase & Very good & Increase & Very good \\
\hline Risk & Yes & No & No & Yes & No \\
\hline
\end{tabular}

\section{5. МтвAC}

Table 1.Comparision on Different Model

A term "Mutual Trust" means a reliable interaction among user and cloud service node with considering their behavior and trust. Access control is an important measure to ensure the security of cloud. It is a policy or procedure that allows, denies or restricts access to a system. Access control may also identify user attempting to access the system unauthorized.

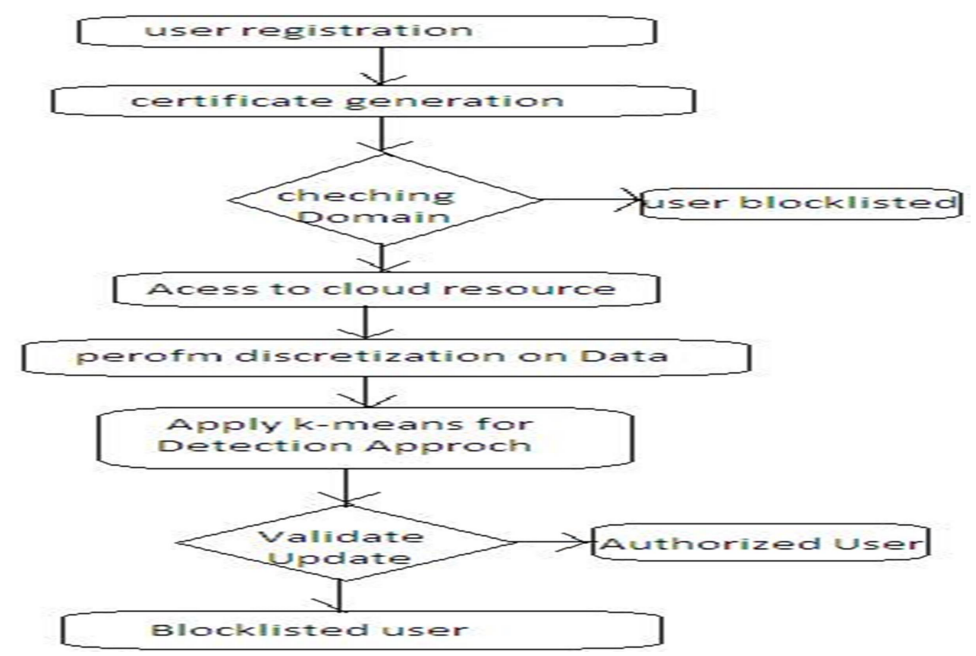

Figure1. Mutual trust structure

The Algorithm of MTBAC:

User request valid or not checked by AAC

$>$ AAC checks the user's behavior

Read the users request and assign cloud node to handle the request in queue

Select the best service node in the node queue and give the service access right to user

Service provide to the user and updates users trust degree 


\subsection{ACO Algorithm}

An ant street is the nature of ant, which is very noticeable thing and we can see it in real world. Ant colony optimization is meta heuristic in which is a colony of artificial ants cooperates in finding good solutions. The ant moves dynamically for searching a food is also called as destination of ant to find shortest path between their also called as the source of ant once an ant has built a solution, or while the solution is being built, the ant evaluates the solution with the pheromone deposit that results in good-quality solution can only emerge as the result of the collective interaction among the ant .when the ant searching for a food then they deposit a pheromone on their path and other ant follows it. if the path is shortest then other ant also follow the same path. Otherwise choose the path to reach destination.

\subsection{K -Means Algorithms}

The grouping is done by minimizing the sum of squares distance between data and corresponding cluster centroid. Thus, the purpose of K-mean clustering is to group the data, if the data is lesser than the number of cluster then assign to the data as the centroid of cluster, every cluster having a unique cluster number. when the data is more than the cluster then we measure the distance between centroid and minimum distance all these data are belonging to the cluster that has less distance from the data $\mathrm{K}$, it is number of cluster we want to find and cluster centroid represent the position in centers of the cluster to initialize the cluster centroid choose $\mathrm{K}$ training samples randomly, and set the cluster centroids to be equal to the values of these $\mathrm{K}$ samples.

\subsection{Particle swarm optimization (PSO)}

PSO has become popular due to its simplicity and its effectiveness in wide range of application with low computational cost. PSO, we used the heuristic to minimize the total cost of execution of application workflows on Cloud Computing environment. PSO has fast speed, but low convergence accuracy. Scheduling Algorithm (SA) has strong commonality, easy to achieve, but long calculation, low efficient, and SA is easy to sink into local optima with serial search [13].

\subsection{Artificial bee colony (ABC) algorithm}

In $\mathrm{ABC}$ model, there are three kinds of honey bee to search food sources, which include scout bees search for food source randomly, employed bees search around the food source and share food information to the onlooker bees, and onlooker bees calculate the fitness and select the best food source. In the nature, bees can extend themselves over long distances in multiple directions. $\mathrm{ABC}$ optimization algorithm which can also solve the Virtual machine scheduling management under the environmental changing of the number of VMs and requests on Cloud computing. Even in changing environment, Cloud computing needs to be operated in a stable system. Therefore, ABC algorithm is suitable for Cloud computing environment because the algorithm is able to effectively utilize the increased system resources and reduce makespan. Its performance is more prominent in scalability [14].

\section{ConClusion And Future Enhancement}

In existing system only password authentication and Role based access control system (RBAC) is used for cloud security but still the intruder gets the access to data by false authentication to the system. For solving this issues use the concept of ACO (Ant Colony Optimization) with Kmeans algorithm and consider the user behavior, once the user get access into the system the $\mathrm{ACO}$ with $\mathrm{K}$-means is used to detect the user update is relevant or not using discretization process. The main difference between RBAC and TRBAC is that the TRBAC consider the user 
International Journal of Information Sciences and Techniques (IJIST) Vol.6, No.1/2, March 2016 trust degree and validate it over time. Access control is a mechanism which can enhance the security of Cloud Computing. It does not check only that the user who logged in is authorized person or not. It also prevents unauthorized users to log in to the system or a cloud environment. it can also prevent the unauthorized activity of authorized user in the cloud environment and also track the behavior of each user. The purpose of this approach by analysis started with identifying the activities of each user and update, the contents are identified as relevant or irrelevant. The level of irrelevance of the content provided by user is use for updating the malicious user.

\section{REFERENCES}

[1] Abdul Raouf Khan, "Access Control in Cloud Computing Environment”, ARPN Journal of Engineering and Applied Science, Volume-7,No.-5, 2012

[2] Guoyuan Lin, Yuyu Bie and Min Lei, "Trust Based Access Control Policy in Multi domain of Cloud Computing”, Journal Computers, Vol.8, No.5,pp.1357 -1366, 2013.

[3] Guoyuan Lin, Yuvu Bie, Danru Wangan Minlei, "MTBAC: A Mutual Trust Based Access Control Model in Cloud Computing", IEEE Journals and Magazines, Volume-11, Issue-4,April-2014.

[4] Guoyuan Lin, Yuyu Bie and MinLei, "Trust Based Access Control Policy in Multi-domain of Cloud Computing", Journal of Computers, Vol.8,No.5, pp.1357-1366, 2013.

[5] Gurmamat Helil, Mucheol Kimand, Sangyong Han "Trust and Risk based Access Control and Access Control Constraints", KSII Transaction On Internet and Information Systems VOL.5, NO.11, November 2011.

[6] Nuno Sontos, Krishna P. Gummadi, Rodrigo Rodrigues "Towards Trusted Colud Compting" article 2009

[7] Mustapha Ben Saidi, Abderrahim Marzouk, "Access Control Protocol for Cloud Systems Based On the Model TorBAC" ,International Journal of soft Computing and Engineering (IJSCE)" ,ISSN: 2231-2307,Volume-2,Issue-5, November-2012.

[8] S.O. Kuyoro, F. Ibikunle and O.Awodele, "Cloud Computing Security Issues and Challenges", International Journal of Computer Networks (IJCN), Volume3, Issue 5, pp. 247-255, 2011.

[9] Zuzana Priscakova and Ivana Rabova, "Model of Solutions for Data Security In Cloud Computing", International Journal of Computer Science, Engineering and Information Technology (IJCSEIT), Vol.3, No.3, pp. 9-19, 2013.

[10] S. Kamara and K. Lauter, "Cryptographic cloud storage," in Proceedings of the 14th international conference on Financial cryptography and data security, pp. 1361-49, 2010.

[11] C. Hota, S. Sanka, M. Rajarajan, S. Nair, "Capability based Cryptographic Data Access Control in Cloud Computing", in International Journal of Advanced Networking and Applications, Volume 01, Issue 01, 2011.

[12] Trusted Computing Group, https:/ www. trustedcomputinggroup.org

[13] Shaobin Zhan, Hongying Huo "Improved PSO-based Task Scheduling Algorithm in Cloud Computing” Journal of Information \& Computational Science 9: 13 (2012) 3821-3829

[14] B. Kruekaew and W. Kimpan, "Virtual Machine Scheduling Management on Cloud Computing Using Artificial Bee Colony" Proceedings of the International Multi Conference of Engineers and Computer Scientists 2014 Vol I, IMECS 2014, March 12 - 14, 2014, Hong Kong

\section{Authors}

I am Payal Buha currently pursuing M.Tech (Cyber Security) from Raksha Shakti University, Ahmedabad, I am working for implementing Cloud Computing Mechanism for Enhancing: MTBAC , I have selected This topic on my dissertation work

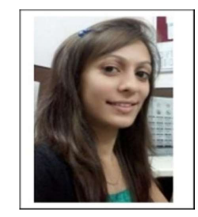

